Revista de Matemática: Teoría y Aplicaciones 2002 9(2) : 39-50

CIMPA - UCR - CCSS ISSN: 1409-2433

\title{
PREFERENCE STRUCTURES, CHOICE FUNCTIONS AND THEIR LOGICAL FORM
}

\author{
María Isabel RodríGuez-Galiano* Jacinto González-Pachón*
}

Received: 15 Nov 2001

\begin{abstract}
Binary relations and choice functions are two different tools for describing DM preferences on a finite set of alternatives. Normal choice functions are the link between these two tools. These choice functions are easily identified using the logical form. In this paper, we analyse normal choice functions from the viewpoint of their logical form.
\end{abstract}

Keywords: Binary relation, choice function, logical form.

\section{Resumen}

Las relaciones binarias y las funciones de elección constituyen dos herramientas diferentes para describir las preferencias del Decisor en un conjunto finito de alternativas. Las funciones de elección normales representan el puente entre estas dos herramientas. Estas funciones de elección se identifican fácilmente utilizando su forma lógica. En este artículo, analizamos las funciones de elección normales a través de su forma lógica.

Palabras clave: Relación binaria, función de elección, forma lógica.

Mathematics Subject Classification: 90A05, 90A06.

\section{Introduction}

Let us suppose a preference modelling problem in which the Decision Maker (DM) has to make a choice from a finite set of alternatives, $\Omega$. Binary relations and choice functions define two different tools for describing DM preferences in such problems.

\footnotetext{
*Dpto. Inteligencia Artificial, Facultad de Informática, Universidad Politécnica de Madrid, Madrid, Spain. E-Mail: irodriguez@fi.upm.es
} 
A decision-making process based on pairwise comparisons introduces a relative evaluation of every pair of alternatives, modelled by a binary relation, $R$

$$
x R y \text { if and only if " } x \text { is at least as good as } y \text { ". }
$$

Three binary relations can be defined from this reflexive binary relation: strict preference, indifference and incomparability. These binary relations are known as the Preference Structure associated with $R$.

In a general choice situation, the DM has to choose from a subset, $X \subseteq \Omega$. This leads to the choice set $C(X)$. The function connecting $(X, C(X))$ is known as a choice function. An obvious way of structuring a choice function is to list the choices made by the DM from each subset of alternatives. However, the logical form provides a shorter form for representing this choice function (Makarov et al., 1987). The logical form and other necessary concepts, will be presented in Section 2.

The Normal Choice Function concept represents a "bridge" between binary relations and choice functions. A normal choice function is a function $C$ that can be represented by a binary relation as follows:

$$
C(X)=\{x \in X / \forall y \in X \quad x R y\} \quad \forall X \subseteq \Omega
$$

Normal choice functions can be easily identified using the logical form.

The purpose of this paper is to explore new aspects of logical forms in a decisionmaking context. We first give the translation of the logical form to the language of choice functions in the case of a normal choice function. In addition, we establish a result by means of which the logical form of a normal choice function can be built from the binary relation that generating this function, and we analyse the relation between the preference structure associated with a binary relation $R$ and the choice function generated by $R$. This will be addressed in Section 3 .

For many applications, it is important to consider pairwise choice, that is, the values of a choice function on sets of two elements (Herzberger, 1973). In Section 4, we study the choice function when restricted to subsets of one and two elements. Because of the importance of the normal choice function, we introduce the normalizable concept to mean restricted choice functions that can be extended to a normal choice function, and then we establish when this is possible. Finally, some conclusions are given in Section 5.

\section{Basic definitions}

Let $\Omega$ be a finite set of alternatives in a decision-making problem, $\Omega=\left\{x_{1}, \ldots, x_{n}\right\}$. Every binary relation $R$ on $\Omega$ can be represented by a matrix

$$
A(R)=\left(a_{i j}(R)\right)_{i, j=1, \ldots, n}
$$

where

$$
a_{i j}(R)=\left\{\begin{array}{ll}
1 & \text { if }\left(x_{i}, x_{j}\right) \in R \\
0 & \text { if }\left(x_{i}, x_{j}\right) \notin R
\end{array} i, j=1, \ldots, n\right.
$$

or a directed graph $G(R)=(\Omega, R)$, where $\Omega$ is the set of vertices and $R$ is the set of arcs.

Let $R$ be a reflexive binary relation on $\Omega$, representing the relation "at least as good as". Taking $R$, let us define the relations $P, I$ and $N$ as follows (Roubens and Vincke, 1985): 
- $x P y$ iff $x R y$ and $y \bar{R} x$.

- $x I y$ iff $x R y$ and $y R x$.

- $x N y$ iff $x \bar{R} y$ and $y \bar{R} x$.

$P$ is called strict preference relation and is the subset of pairs $(x, y)$ such that the statement " $x$ is preferred to $y$ " is true.

$I$ is called indifference relation and is the subset of pairs $(x, y)$ such that " $x$ is indifferent to $y "$.

$N$ is the incomparability or noncomparability relation, and it is formed by the pairs $(x, y)$ such that " $x$ is not comparable to $y$ " (the individual is unable to compare the elements $x$ and $y$ ).

Definition 1: The triplet of relations $\{P, I, N\}$ will be called preference structure.

Now, we give the formal definition of a choice function.

Definition 2: A choice function $C$ on $\Omega$ is a function $C: \mathcal{P}(\Omega) \longrightarrow \mathcal{P}(\Omega)$ such that $\forall X \subset \Omega C(X) \subset X$.

$C(X)$ is the set of alternatives chosen by the DM from $X$.

An alternative $x$ is termed singular in respect of $C$ if $C(\{x\})=\varnothing$ and nonsingular if $C(\{x\})=\{x\}$ (Aizerman, 1985). Furthermore, a choice function $C$ is called reflexive if all the alternatives are nonsingular, i.e., $C(\{x\})=\{x\} \forall x \in \Omega$.

The most straightforward form of representing $C$ is to list the choices made by the DM from each subset of alternatives. However, Boolean algebra can be used to get a shorter form of expressing a choice function: the logical form.

Each subset $X$ of $\Omega$ is associated with the vector $\beta(X)=\left(\beta_{1}(X), \ldots, \beta_{n}(X)\right)$ of Boolean variables, where

$$
\beta_{i}(X)=\left\{\begin{array}{ll}
1 & \text { if } x_{i} \in X \\
0 & \text { if } x_{i} \notin X
\end{array} \forall i=1, \ldots, n\right.
$$

Clearly, $\beta(\Omega)=(1, \ldots, 1)$ and $\beta(\varnothing)=(0, \ldots, 0)$.

Let $C$ be a choice function defined on $\Omega$.

Definition 3: The logical form of the choice function $C(\operatorname{LFC}(C))$ is the family of Boolean functions ${ }^{1}\left(f_{1}, \ldots, f_{n}\right)$ of $n-1$ variables, such that

$$
\beta_{i}(X) \wedge f_{i}(\beta(X))=1 \Longleftrightarrow x_{i} \in C(X)
$$

where

$$
\begin{aligned}
f_{i}(\beta) & =f_{i}\left(\beta_{1}, \ldots, \beta_{i-1}, \beta_{i+1}, \ldots, \beta_{n}\right) \text { for } i \neq 1, n \\
f_{1}(\beta) & =f_{1}\left(\beta_{2}, \ldots, \beta_{n}\right) \\
f_{n}(\beta) & =f_{n}\left(\beta_{1}, \ldots, \beta_{n-1}\right)
\end{aligned}
$$

\footnotetext{
${ }^{1}$ A Boolean function $f$ is a mapping $f: B_{2}^{m}=\underbrace{B_{2} \times \ldots \times B_{2}}_{m} \rightarrow B_{2}$, where $B_{2}=\{0,1\}$.
} 
If $x_{i} \notin X$, it makes no sense to check whether or not $x_{i}$ is chosen from the subset $X$. Hence, this definition is simplified if we assume that implicitly $\beta_{i}=1$ in $f_{i}(\beta)$. Thus, we have that

$$
x_{i} \in C(X) \Longleftrightarrow f_{i}(\beta(X))=1
$$

(if $\beta_{i}=0$, we know that $x_{i} \notin C(X)$, because $\left.x_{i} \notin X\right)$.

A choice function can be associated to every binary relation, however, the inverse is not true; i.e., there are choice functions that cannot be represented by a binary relation.

Normal choice functions represent the link between binary relations and choice functions.

Definition 4: A choice function is normal if there is a binary relation $R$ such that $C=C_{R}$, where

$$
C_{R}(X)=\{x \in X / \forall y \in X \quad x R y\} \quad \forall X \subseteq \Omega
$$

In this case, we say that the choice function $C$ is generated by the binary relation $R$.

\section{Normal Choice Function}

In this section, a normal choice function and its associated preference are connected by the logical form.

The following result connects binary choices with a general one.

Theorem 1: (Rodríguez-Galiano and González-Pachón, 2001) Let C be a choice function on $\Omega$. $C$ is normal iff

$$
C(X)=\bigcup_{x \in X}\left[\bigcap_{y \in X} C(\{x, y\})\right] \forall X \subseteq \Omega .
$$

This result describes what the choice functions that can be represented by a binary relation are like: they are the functions where the selection criteria are summarized in pairwise comparisons (including the unitary comparisons).

The logical form of a choice function provides an easy method for determining when there is an associated binary relation. The following result is useful for identifying normal choice functions.

Theorem 2: (Makarov et al., 1987) A choice function $C$ on $\Omega=\left\{x_{1}, x_{2}, \ldots, x_{n}\right\}$ is normal iff there exists a partition of the set $J=\{1, \ldots, n\}$ into the subsets $I, I_{0}, I_{1}$ (any one of them might be empty) such that

1. $\forall i \in I \quad \exists J_{i} \subset J, J_{i} \neq \varnothing$ s.t. $f_{i}=\bigwedge_{j \in J_{i}} \bar{\beta}_{j}$.

2. $\forall i \in I_{0} \quad f_{i} \equiv 0$.

3. $\forall i \in I_{1} \quad f_{i} \equiv 1$. 
Thus, a choice function $C$ on $\Omega$ is a normal choice function iff the Boolean expression of the $f_{i}$ 's that formed its logical form are 0,1 or a conjunction of refuted terms, i.e., $f_{i} \in\left\{0,1, \bigwedge_{j \in J_{i}} \bar{\beta}_{j}\right\} \forall i$

The interpretation of the previous theorem is as follows: a choice function is generated by a binary relation iff, given an element $x_{i} \in \Omega$, one of the following affirmations is true:

1. $x_{i}$ is chosen whenever none of the elements $x_{i_{1}}, \ldots, x_{i_{k}}\left(\right.$ if $\left.J_{i}=\left\{i_{1}, \ldots, i_{k}\right\}\right)$ are in the proposed subset $X$, i.e., it is chosen if $\left\{x_{i_{1}}, \ldots, x_{i_{k}}\right\} \bigcap X=\varnothing$.

$$
x_{i} \in C(X) \Longleftrightarrow\left(x_{i_{1}} \notin X, x_{i_{2}} \notin X, \ldots, x_{i_{k}} \notin X\right)
$$

2. $x_{i}$ is never chosen, i.e., $x_{i} \notin C(X) \quad \forall X \subseteq \Omega$.

3. $x_{i}$ is always chosen, i.e., $x_{i} \in C(X) \quad \forall X \subseteq \Omega$ with $x_{i} \in X$.

As mentioned above, a choice function can be represented in a shorter and more manageable manner using the logical rather than explicit form. Given a binary relation $R$, it would be useful to get the logical form of the choice function it generates, without having to determine this explicitly. Let us see how the choice function can be calculated from the matrix $A(R)$ or, equivalently, from the graph $G(R)$.

Proposition 1: Let $R$ be a binary relation represented by the matrix $A(R)$ and let $L F C\left(C_{R}\right)=\left(f_{1}, f_{2}, \ldots, f_{n}\right)$ be the logical form of the choice function generated by $R$. Then

- $f_{i}=0 \Longleftrightarrow a_{i i}=0$.

- $f_{i}=1 \Longleftrightarrow a_{i j}=1 \quad \forall j$

- $f_{i}=\bigwedge_{j \in J_{i}} \bar{\beta}_{j}$ with $J_{i}=\left\{h / a_{i h}=0\right\} \Longleftrightarrow a_{i i}=1$ and $\exists j \neq i$ s.t. $a_{i j}=0$.

\section{Proof:}

$$
\begin{aligned}
f_{i}= & 0 \Longleftrightarrow a_{i i}=0 \\
& a_{i i}=0 \Leftrightarrow x_{i} \bar{R} x_{i} \Leftrightarrow x_{i} \notin C_{R}(X) \forall X \subseteq \Omega \Leftrightarrow f_{i}=0 . \\
f_{i}= & 1 \Longleftrightarrow a_{i j}=1 \quad \forall j \\
& a_{i j}=1 \quad \forall j \Leftrightarrow x_{i} R x_{j} \quad \forall j \Leftrightarrow x_{i} \in C_{R}(X) \forall X \subseteq \Omega \Leftrightarrow f_{i}=1 . \\
f_{i}= & \bigwedge_{j \in J_{i}} \bar{\beta}_{j} \text { with } J_{i}=\left\{h / a_{i h}=0\right\} \Longleftrightarrow a_{i i}=1 \text { and } \exists j \neq i \text { s.t. } a_{i j}=0 \\
& (\Longrightarrow) \text { If } f_{i}=\bigwedge_{j \in J_{i}} \bar{\beta}_{j}, \text { we have that } J_{i} \neq \varnothing \text { and then } \exists k \neq i \text { such that } x_{i} \bar{R} x_{k} \text {. Thus, } \\
& a_{i k}=0 . \\
& a_{i i}=1 \text { since if } a_{i i}=0, f_{i}=0, \text { which is a contradiction. } \\
& (\Longleftarrow) a_{i i}=1 \Leftrightarrow x_{i} R x_{i} . \\
& \text { Let } J_{i}=\left\{h / a_{i h}=0\right\} . \text { Since } \exists k \neq i \text { s.t. } a_{i k}=0, J_{i} \text { is not empty. Moreover, }
\end{aligned}
$$

$$
a_{i j}=0 \forall j \in J_{i} \Leftrightarrow x_{i} \bar{R} x_{j} \forall j \in J_{i} .
$$


Given $X \subset \Omega$, we have that $x_{i} \in C_{R}(X) \Leftrightarrow x_{j} \notin X \quad \forall j \in J_{i} \Leftrightarrow \beta_{j}(X)=0 \quad \forall j \in J_{i}$. Then, taking into account that $x_{i} \in C_{R}(X) \Leftrightarrow f_{i}(\beta(X))=1$, we get $f_{i}=\bigwedge_{j \in J_{i}} \bar{\beta}_{j}$.

Obviously, it is enough to observe the ith row of matrix $A(R)$ to get $f_{i}$.

Example 1: Let $\Omega=\left\{x_{1}, x_{2}, x_{3}, x_{4}\right\}$ and the binary relation $R$ be defined by the following matrix:

$$
A(R)=\left(\begin{array}{llll}
1 & 1 & 0 & 0 \\
1 & 1 & 1 & 1 \\
1 & 0 & 1 & 1 \\
0 & 0 & 0 & 1
\end{array}\right)
$$

Applying the previous proposition, we get that the logical form of the choice function generated by $R$ is:

$$
\begin{aligned}
& f_{1}\left(\beta_{2}, \beta_{3}, \beta_{4}\right)=\bar{\beta}_{3} \bar{\beta}_{4} \\
& f_{2}\left(\beta_{1}, \beta_{3}, \beta_{4}\right)=1 \\
& f_{3}\left(\beta_{1}, \beta_{2}, \beta_{4}\right)=\bar{\beta}_{2} \\
& f_{4}\left(\beta_{1}, \beta_{2}, \beta_{3}\right)=\bar{\beta}_{1} \bar{\beta}_{2} \bar{\beta}_{3}
\end{aligned}
$$

That is,

$$
\operatorname{LFC}\left(C_{R}\right)=\left(\bar{\beta}_{3} \bar{\beta}_{4}, 1, \bar{\beta}_{2}, \bar{\beta}_{1} \bar{\beta}_{2} \bar{\beta}_{3}\right)
$$

From both the explicit and logical form of the normal choice function $C$, we can get the binary relations that form the preference structure.

First, let us see the relation between preference structure and a normal choice function.

Given a reflexive binary relation $R$, let us see what $C_{R}(X)$ indicates for each subset $X$ of $\Omega$.

- If $C_{R}(X)=\varnothing$ with $|X|=2$, the elements of $X$ are not comparable (then we get the relation $N$ of the preference structure $\{P, I, N\}$ associated with $R$ ).

- If $C_{R}(X)=\varnothing$ with $|X|>2$, the elements of $X$ are either not comparable or indifferent (there are non-comparable elements, and there could be indifferent elements), or some elements are preferred to others but not to all elements.

- If $C_{R}(X)=X$ the elements of $X$ are indifferent to each other (thus, we get the relation $I)$.

- If $C_{R}(X)=\left\{x_{i}\right\}$ with $x_{i} \in X$, then $x_{i}$ is the element preferred to all elements of the subset $X$, i.e., from subset $X$, we choose the alternative $x_{i}$ (then we get the relation $P)$.

- If $C_{R}(X)=X_{i}$ with $X_{i} \subset X$ and $1<\left|X_{i}\right|<|X|$, the elements of $X_{i}$ are indifferent to each other, but they are preferred to the elements of $X \backslash X_{i}$.

Thus, we can get the preference structure $\{P, I, N\}$ from the choice function $C_{R}$, as follows:

Consider $x, y \in \Omega, x \neq y$ 
- $x P y$ iff $C_{R}(\{x, y\})=\{x\}$.

- $x I y$ iff $C_{R}(\{x, y\})=\{x, y\}$.

- $x N y$ iff $C_{R}(\{x, y\})=\varnothing$.

Moreover, $x I x \quad \forall x \in \Omega$.

Example 2: Let us consider the binary relation $R$ of the previous example. $R$ generates the choice function shown in Table 1 . Then, the preference structure associated with $R$ is

Table 1: $C_{R}$

\begin{tabular}{|c|c|}
\hline $\mathrm{X}$ & $C_{R}(X)$ \\
\hline$x_{1}$ & $x_{1}$ \\
\hline$x_{2}$ & $x_{2}$ \\
\hline$x_{3}$ & $x_{3}$ \\
\hline$x_{4}$ & $x_{4}$ \\
\hline$x_{1}, x_{2}$ & $x_{1}, x_{2}$ \\
\hline$x_{1}, x_{3}$ & $x_{3}$ \\
\hline$x_{1}, x_{4}$ & $\varnothing$ \\
\hline$x_{2}, x_{3}$ & $x_{2}$ \\
\hline$x_{2}, x_{4}$ & $x_{2}$ \\
\hline$x_{3}, x_{4}$ & $x_{3}$ \\
\hline$x_{1}, x_{2}, x_{3}$ & $x_{2}$ \\
\hline$x_{1}, x_{2}, x_{4}$ & $x_{2}$ \\
\hline$x_{1}, x_{3}, x_{4}$ & $x_{3}$ \\
\hline$x_{2}, x_{3}, x_{4}$ & $x_{2}$ \\
\hline$x_{1}, x_{2}, x_{3}, x_{4}$ & $x_{2}$ \\
\hline
\end{tabular}

given below.

$$
\begin{aligned}
P & =\left\{\left(x_{2}, x_{3}\right),\left(x_{2}, x_{4}\right),\left(x_{3}, x_{1}\right),\left(x_{3}, x_{4}\right)\right\} \\
I & =\left\{\left(x_{1}, x_{2}\right),\left(x_{2}, x_{1}\right),\left(x_{1}, x_{1}\right),\left(x_{2}, x_{2}\right),\left(x_{3}, x_{3}\right),\left(x_{4}, x_{4}\right)\right\} \\
N & =\left\{\left(x_{1}, x_{4}\right),\left(x_{4}, x_{1}\right)\right\}
\end{aligned}
$$

If, instead of the explicit form, we have the logical form of $C_{R}$, we get the following result.

Proposition 2: Consider $\Omega=\left\{x_{1}, \ldots, x_{n}\right\}$ and let $R$ be a reflexive binary relation on $\Omega$. If $\operatorname{LFC}\left(C_{R}\right)=\left(f_{1}, \ldots, f_{n}\right)$ is the logical form of the choice function generated by $R$ and $\{P, I, N\}$ is the preference structure associated with $R$, then

$\forall i, j \in J=\{1, \ldots, n\}, i \neq j$ 
- $x_{i} P x_{j}$ iff $\exists L_{j} \subset J$ with $i \in L_{j}$ s.t. $f_{j}=\bigwedge_{l \in L_{j}} \bar{\beta}_{l}$ and $\left(f_{i}=1\right.$ or $\exists L_{i} \subset J$ with $j \notin L_{i}$ and $L_{i} \neq \varnothing$ s.t. $f_{i}=\bigwedge_{l \in L_{i}} \bar{\beta}_{l}$ ) (in an abridged form, we write:

$$
\left.f_{i} \in\left\{1, \bigwedge_{l \in L_{i}} \bar{\beta}_{l} \text { with } j \notin L_{i}\right\} \quad \text { and } f_{j}=\bigwedge_{l \in L_{j}} \bar{\beta}_{l} \text { with } i \in L_{j}\right) \text {. }
$$

- $x_{i} I x_{j}$ iff $f_{i} \in\left\{1, \bigwedge_{l \in L_{i}} \bar{\beta}_{l}\right.$ with $\left.j \notin L_{i}\right\} \quad$ and $f_{j} \in\left\{1, \bigwedge_{l \in L_{j}} \bar{\beta}_{l}\right.$ with $\left.i \notin L_{j}\right\}$.

- $x_{i} N x_{j}$ iff $f_{i}=\bigwedge_{l \in L_{i}} \bar{\beta}_{l}$ with $j \in L_{i}$ and $f_{j}=\bigwedge_{l \in L_{j}} \bar{\beta}_{l}$ with $i \in L_{j}$.

Proof: Since $R=P \cup I$ is reflexive, $f_{i} \neq 0, \forall i=1, \ldots, n$ in the logical form of $C_{R}$, and SO

$$
f_{i} \in\left\{1, \bigwedge_{j \in J_{i}} \bar{\beta}_{j}\right\}, \forall i=1, \ldots, n .
$$

$x_{i} P x_{j}$ iff $C_{R}\left(\left\{x_{i}, x_{j}\right\}\right)=\left\{x_{i}\right\}$ iff $x_{i}$ is chosen from subset $\left\{x_{i}, x_{j}\right\}$ and $x_{j}$ is not chosen when $x_{i}$ is in the proposed subset (see Theorem 1 ).

Let us see how these last two affirmations are expressed in terms of the logical form:

1. $x_{i}$ is chosen from subset $\left\{x_{i}, x_{j}\right\}$

There are two possible cases.

(a) $x_{i}$ is always chosen. This happens if and only if $f_{i}=1$.

(b) $x_{i}$ is not always chosen, but it is, at least, chosen from subset $\left\{x_{i}, x_{j}\right\}$ iff $\exists L_{i} \subset J_{i}, L_{i} \neq \varnothing$ s.t. $j \notin L_{i}$ and $f_{i}=\bigwedge_{l \in L_{i}} \bar{\beta}_{l}$.

2. $x_{j}$ is not chosen when $x_{i}$ is in the proposed subset.

In this case, $\exists L_{j} \subset J_{i}, L_{j} \neq \varnothing$ with $i \in L_{j}$ s.t. $f_{j}=\bigwedge_{l \in L_{j}} \bar{\beta}_{l}$.

For relations $I$ and $N$ we have, $x_{i} I x_{j}$ iff $C_{R}\left(\left\{x_{i}, x_{j}\right\}\right)=\left\{x_{i}, x_{j}\right\}$ iff $x_{i}$ is chosen from subset $\left\{x_{i}, x_{j}\right\}$ and $x_{j}$ is chosen from subset $\left\{x_{i}, x_{j}\right\}$.

$x_{i} N x_{j}$ iff $C_{R}\left(\left\{x_{i}, x_{j}\right\}\right)=\varnothing$ iff $x_{i}$ is not chosen when $x_{j}$ is in the proposed subset and $x_{j}$ is not chosen when $x_{i}$ is in the subset in question.

Then, applying the above results for the preference relation $P$ to relations $I$ and $N$, the proposition is proved.

Example 3: Consider the same choice function $C_{R}$ as in the previous examples, whose logical form is (see Example 1):

$$
\operatorname{LFC}\left(C_{R}\right)=\left(\bar{\beta}_{3} \bar{\beta}_{4}, 1, \bar{\beta}_{2}, \bar{\beta}_{1} \bar{\beta}_{2} \bar{\beta}_{3}\right)
$$

Then,

$$
L_{1}=\{3,4\}, L_{3}=\{2\}, L_{4}=\{1,2,3\}
$$


We get $P$ by observing that $f_{2}=1$ and then searching for $x_{j} \in \Omega$ such that $x_{2} P x_{j}$, i.e., the elements for which $f_{j}=\bigwedge_{l \in L_{j}} \bar{\beta}_{l}$ with $2 \in L_{j}$. We have that $2 \in L_{3} \cap L_{4}$, so that $x_{2} P x_{3}$ and $x_{2} P x_{4}$.

For $f_{3}=\bar{\beta}_{2}$, we have that $1,4 \notin L_{3}$. Thus, we focus on $f_{1}$ and $f_{4}$. Since $3 \in L_{1} \cap L_{4}$, we get that $x_{3} P x_{1}$ and $x_{3} P x_{4}$.

For $I$, we have that $f_{2}=1$ and $2 \notin L_{1}$, so that $x_{2} I x_{1}$ and $x_{1} I x_{2}$. Furthermore, we know that $I$ is reflexive.

For $N$, we observe that $f_{1}=\bar{\beta}_{3} \bar{\beta}_{4}$ and $1 \in L_{4}$, therefore $x_{1} N x_{4}$ and $x_{4} N x_{1}$.

Thus,

$$
\begin{aligned}
P & =\left\{\left(x_{2}, x_{3}\right),\left(x_{2}, x_{4}\right),\left(x_{3}, x_{1}\right),\left(x_{3}, x_{4}\right)\right\} \\
I & =\left\{\left(x_{1}, x_{2}\right),\left(x_{2}, x_{1}\right),\left(x_{1}, x_{1}\right),\left(x_{2}, x_{2}\right),\left(x_{3}, x_{3}\right),\left(x_{4}, x_{4}\right)\right\} \\
N & =\left\{\left(x_{1}, x_{4}\right),\left(x_{4}, x_{1}\right)\right\}
\end{aligned}
$$

which is the same as we got before (in Example 2).

\section{Restriction of Choice Functions to Pairwise Comparison}

Now, we study choice functions when the DM has to make a choice in subsets of one or two elements.

Let $C_{c p}$ be a choice function known only in $\Omega$ subsets, whose cardinal is smaller or equal than 2 .

Definition 5: The restricted choice function $C_{c p}$ is normalizable if there is a binary relation $R$ such that $C_{c p}(X)=C_{R}(X) \forall X \subseteq \Omega$ with $\operatorname{card}(X)=1,2$.

We use the term normalizable, because if $\exists R$ such that $C_{c p}(X)=C_{R}(X) \quad \forall X \subseteq \Omega$ with $|X|=1,2$, we can extend the choice function $C_{c p}$ to the other subsets so that $C_{c p}(X)=C_{R}(X) \forall X \subseteq \Omega$, getting a normal choice function.

Proposition 3: $C_{c p}$ is normalizable iff

$$
C_{c p}(\{x\})=\varnothing \Rightarrow x \notin C_{c p}(\{x, y\}) \forall y \neq x
$$

Proof: According to Theorem 1, we have that a choice function is normal iff

$$
C(X)=\bigcup_{x \in X} \bigcap_{y \in X} C(\{x, y\}) \forall X \subseteq \Omega
$$

In particular, it should be true for the subsets of cardinal smaller or equal than two. Thus, we have that $C_{c p}$ is normalizable iff

$$
C_{c p}(X)=\bigcup_{x \in X} \bigcap_{y \in X} C_{c p}(\{x, y\}) \forall X \subseteq \Omega \text { with } \operatorname{card}(X)=1,2 .
$$


iff $C_{c p}(\{x, y\})=\bigcup_{x \in X} \bigcap_{y \in X} C_{c p}(\{x, y\})=\left(C_{c p}(\{x\}) \cap C_{c p}(\{x, y\})\right) \cup\left(C_{c p}(\{y\}) \cap C_{c p}(\{x, y\})\right)$. Therefore, it is clear that

$$
C_{c p} \text { is normalizable iff } C_{c p}(\{x\})=\varnothing \Rightarrow x \notin C_{c p}(\{x, y\}) \forall y \neq x \text {. }
$$

Thus, if the DM is coherent and he/she rejects an alternative $x$, the problem would be equivalent to the selection problem in $\Omega \backslash\{x\}$. That is, we can remove singular alternatives from the problem. Nevertheless, we find that this is not always possible, since the presence of such an alternative can influence the choice of other alternatives (Bauman, 1977). Only if the alternative does not affect to the selection process can it be excluded.

Moreover, we can extend the restricted choice function $C_{c p}$ to the normal choice function $C$, as follows:

$$
\begin{aligned}
& C(X)=C_{c p}(X) \forall X \subseteq \Omega \text { s.t. }|X| \leq 2 \\
& C(X)=\bigcup_{x \in X} \bigcap_{y \in X \backslash\{x\}} C(\{x, y\}) \forall X \subseteq \Omega \text { s.t. }|X|>2
\end{aligned}
$$

Obviously, the extension is unique.

Example 4: Consider the restricted choice functions represented in Table 2.

Table 2: Restricted functions

\begin{tabular}{|c|c|c|c|}
\hline$X$ & $C_{c p_{1}}(X)$ & $C_{c p_{2}}(X)$ & $C_{c p_{3}}(X)$ \\
\hline$x_{1}$ & $x_{1}$ & $x_{1}$ & $x_{1}$ \\
\hline$x_{2}$ & $\varnothing$ & $x_{2}$ & $\varnothing$ \\
\hline$x_{3}$ & $x_{3}$ & $x_{3}$ & $x_{3}$ \\
\hline$x_{4}$ & $\varnothing$ & $x_{4}$ & $\varnothing$ \\
\hline$x_{1}, x_{2}$ & $x_{1}, x_{2}$ & $x_{1}, x_{2}$ & $x_{1}$ \\
\hline$x_{1}, x_{3}$ & $x_{1}$ & $x_{1}$ & $x_{1}$ \\
\hline$x_{1}, x_{4}$ & $x_{4}$ & $x_{4}$ & $\varnothing$ \\
\hline$x_{2}, x_{3}$ & $x_{2}$ & $x_{2}$ & $\varnothing$ \\
\hline$x_{2}, x_{4}$ & $x_{4}$ & $x_{4}$ & $\varnothing$ \\
\hline$x_{3}, x_{4}$ & $x_{3}$ & $x_{3}$ & $x_{3}$ \\
\hline
\end{tabular}

$C_{c p_{1}}$ is not normalizable, since $C_{c p_{1}}\left(\left\{x_{2}\right\}\right)=C_{c p_{1}}\left(\left\{x_{4}\right\}\right)=\varnothing$ and

$$
\begin{array}{ll}
x_{2} \in C\left(\left\{x_{2}, y\right\}\right), & y=x_{1}, x_{3} \\
x_{4} \in C\left(\left\{x_{4}, y\right\}\right), & y=x_{1}, x_{2}
\end{array}
$$

However, $C_{c p_{2}}$ and $C_{c p_{3}}$ are normalizable, and their extensions are the choice functions $C_{2}$ and $C_{3}$ respectively, shown in Table 3 . 
Table 3: $C_{2}$ and $C_{3}$

\begin{tabular}{|c|c|c|}
\hline$X$ & $C_{2}(X)$ & $C_{3}(X)$ \\
\hline$x_{1}$ & $x_{1}$ & $x_{1}$ \\
\hline$x_{2}$ & $x_{2}$ & $\varnothing$ \\
\hline$x_{3}$ & $x_{3}$ & $x_{3}$ \\
\hline$x_{4}$ & $x_{4}$ & $\varnothing$ \\
\hline$x_{1}, x_{2}$ & $x_{1}, x_{2}$ & $x_{1}$ \\
\hline$x_{1}, x_{3}$ & $x_{1}$ & $x_{1}$ \\
\hline$x_{1}, x_{4}$ & $x_{4}$ & $\varnothing$ \\
\hline$x_{2}, x_{3}$ & $x_{2}$ & $\varnothing$ \\
\hline$x_{2}, x_{4}$ & $x_{4}$ & $\varnothing$ \\
\hline$x_{3}, x_{4}$ & $x_{3}$ & $x_{3}$ \\
\hline$x_{1}, x_{2}, x_{3}$ & $x_{1}, x_{2}$ & $x_{1}$ \\
\hline$x_{1}, x_{2}, x_{4}$ & $x_{4}$ & $\varnothing$ \\
\hline$x_{1}, x_{3}, x_{4}$ & $\varnothing$ & $\varnothing$ \\
\hline$x_{2}, x_{3}, x_{4}$ & $\varnothing$ & $\varnothing$ \\
\hline$x_{1}, x_{2}, x_{3}, x_{4}$ & $\varnothing$ & $\varnothing$ \\
\hline
\end{tabular}

\section{Conclusions}

The logical form of a choice function is a good tool for representing a choice function in a shorter way. In this paper, we have shown how the logical form of a normal choice function can be obtained directly from the associated binary relation. Moreover, we have shown how the preference structure related to the binary relation related to a choice function can be obtained from the logical form.

If we consider only subsets of cardinal less than or equal to two, it is not always possible to represent the restricted choice function using a binary relation. Provided that the choice is made according to a normal choice function, the problem would be equivalent to the selection problem in the subset of nonsingular alternatives.

\section{Acknowledgement}

Work supported by "Consejería de Educación y Cultura de la Comunidad de Madrid" under the project $07 \mathrm{~T} / 0027 / 2000$.

\section{References}

[1] Aizerman, M.A. (1985) "New problems in the general choice theory", Social Choice and Welfare 2(2): 235-282.

[2] Bauman, YE.V. (1977) "Choice on Graph and in a Criterial Space", Automation and remote control. Simulation of behavior and intelligence: 710-721. (Translated from Avtomatika $i$ Telemekhanica 5: 114-126.) 
[3] Herzberger, H. (1973) "Ordinal preference and rational choice", Econometrica 41: $187-237$.

[4] Makarov, I.M.; Vinogradskaya, T.M.; Rubchinsky, A.A.; Sokolov, V.B. (1987) The Theory of Choice and Decision Making. Mir Publishers Moscow.

[5] Rodríguez-Galiano, M.I.; González-Pachón, J. (2001) "Characterization of certain orders using their associated choice functions", European Journal of Operational Research 132: $619-627$.

[6] Roubens, M.; Vincke, Ph. (1985) Preference Modelling. LNEMS 250, Springer-Verlag. 